\title{
Alignment Measurements of the Microwave Anisotropy Probe (MAP) Instrument in a Thermal/Vacuum Chamber using Photogrammetry
}

\author{
Michael D. Hill ${ }^{* a}$, Acey A. Herrera ${ }^{b}$, J. Allen Crane ${ }^{b}$, Edward A. Packard ${ }^{a}$, Carlos Aviado $^{c}$, \\ Henry P. Sampler ${ }^{\text {a }}$ \\ a Goddard Space Flight Center, Greenbelt, MD \\ ${ }^{\mathrm{b}}$ Swales Aerospace, Inc., Beltsville, MD \\ ${ }^{\mathrm{c}}$ ManTech, Greenbelt, MD
}

\begin{abstract}
The Microwave Anisotropy Probe (MAP) Observatory, scheduled for a late 2000 launch, is designed to measure temperature fluctuations (anisotropy) and produce a high sensitivity and high spatial resolution (< $0.3^{\circ}$ at $90 \mathrm{GHz}$.) map of the cosmic microwave background (CMB) radiation over the entire sky between 22 and $90 \mathrm{GHz}$. MAP utilizes back-to-back Gregorian telescopes to focus the microwave signals into 10 differential microwave receivers, via 20 feed horns. Proper alignment of the telescope reflectors and the feed horns at the operating temperature of $90 \mathrm{~K}$ is a critical element to ensure mission success.

We describe the hardware and methods used to validate the displacement/deformation predictions of the reflectors and the microwave feed horns during thermal/vacuum testing of the reflectors and the microwave instrument. The smallest deformations to be resolved by the measurement system were on the order of \pm 0.030 inches $(0.762 \mathrm{~mm})$.

Performance of these alignment measurements inside a thermal/vacuum chamber with conventional alignment equipment posed several limitations. A photogrammetry ( $P G$ ) system was chosen to perform the measurements since it is a non-contact measurement system, the measurements can be made relatively quickly and accurately, and the photogrammetric camera can be operated remotely.

The hardware and methods developed to perform the MAP alignment measurements using PG proved to be highly successful. The PG measurements met the desired requirements, enabling the desired deformations to be measured and even resolved to an order of magnitude smaller than the imposed requirements. Viable data were provided to the MAP Project for a full analysis of the on-orbit performance of the Instrument's microwave system.
\end{abstract}

KEY WORDS: MAP, photogrammetry, thermal/vacuum, alignment

\subsection{INTRODUCTION}

The Microwave Anisotropy Probe (MAP) Observatory, designed and built by NASA Goddard Space Flight Center in partnership with Princeton University, will measure temperature fluctuations (anisotropy) and produce a high sensitivity and high spatial resolution map of the cosmic microwave background (CMB) radiation over the entire sky between 22 and $90 \mathrm{GHz}$. The CMB radiation is the remnant radiant heat left over from the Big Bang.

MAP will measure the anisotropy of the CMB radiation over the full sky with an angular resolution of at least 0.3 degrees, a sensitivity of $20 \mathrm{uK}$ per 0.3 degree square pixel, and with systematic effects limited to 5 $\mathrm{uK}$ per pixel. MAP will obtain and process differential temperature data, rather than sensing absolute temperatures, to produce a differential temperature map of the sky. This will be accomplished by using two back-to-back optical systems followed by a set of 10 differential microwave receivers and associated signal processing electronics. The Observatory will have a compound scan with a spin rate of $\sim 0.454 \mathrm{rpm}$ and a precession rate of $\sim 1$ revolution per hour. This allows MAP to collect data over a $45^{\circ} \times 180^{\circ}$ swath of the sky every hour. The microwave receivers will cool to $\sim 95 \mathrm{~K}$, while the reflectors will cool to $\sim 40 \mathrm{~K}$. 
Proper alignment of the telescope reflectors and the receiver feed horns at the operating temperature of 90 $\mathrm{K}$ is a critical element to ensure mission success. The instrument components, made of various materials (aluminum, copper, composite, etc.), were designed based on deformations predicted by thermal modeling. In order to ensure that the hardware behaved as predicted at cold operating temperatures, alignment measurements would have to be taken during the thermal/vacuum testing of the prototype and flight hardware. These measurements would have to resolve the deformations of the critical instrument components as the hardware reached it's operating temperature.

We describe the hardware and methods used to validate the displacement/deformation predictions of the reflectors and the microwave feed horns during thermal/vacuum testing of the reflectors and the microwave instrument. The smallest deformations to be resolved by the measurement system were on the order of \pm 0.030 inches $(0.762 \mathrm{~mm})$. The system chosen to perform these alignment measurements was the photogrammetric camera system.

\subsection{MAP Hardware Description}

MAP is essentially a dual-telescope differential microwave receiver. The two telescopes are mounted back-to-back such that each telescope can collect microwave signals simultaneously from different parts of the sky as the Observatory spins about it's axis. The instrument is divided into two receivers, an A-side and a B-side, each side having a telescope and it's associated microwave sensing systems. Differencing assemblies then combine the signals collected by the two sides.

The two major subsystems involved in the PG measurements are the Thermal Reflector System (TRS) and the Microwave System (MS). The TRS and the MS mount onto the spacecraft bus, as shown in the exploded view of the MAP Observatory in Figure 1. The TRS and the MS, when assembled, comprise the Instrument, as shown in Figure 2.

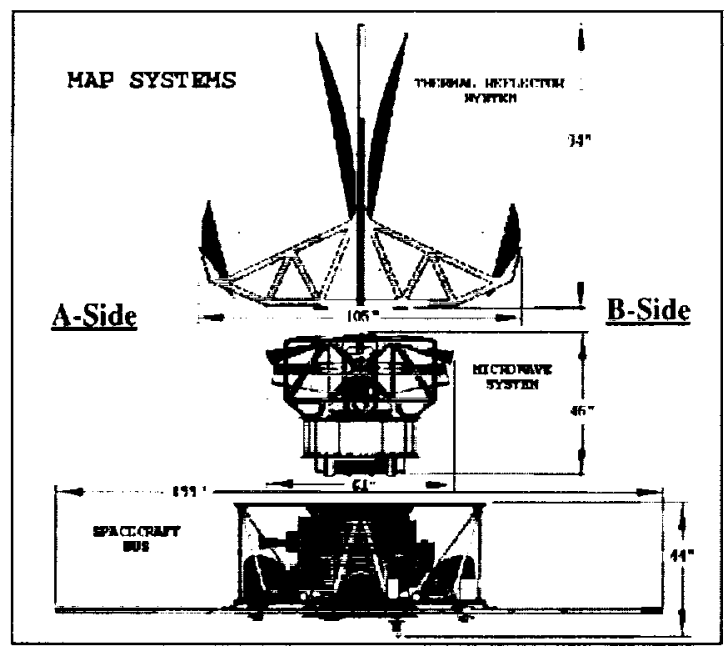

Figure 1 - MAP Systems - Exploded View

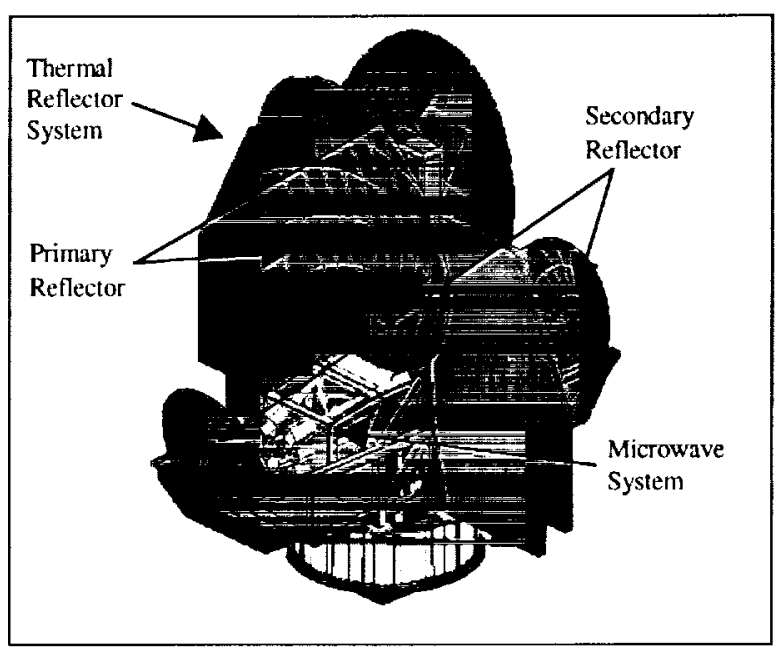

Figure 2 - MAP Instrument - Assembled View

The TRS, shown in Figure 3, consists of a truss structure that is designed to support and maintain alignment of the telescope reflectors, which focus the microwave energy into the MS. The reflectors were manufactured by PCI of Anaheim, California, and are constructed of Korex Honeycomb composite with a vapor deposited aluminum and SiOx coating.

The MS, shown in Figure 4, houses the microwave feed horns and the microwave amplifiers. The MS consists of the Focal Plane Assembly (FPA) and the Receiver/Transceiver Box (RXB). The FPA supports and aligns the 20 feed horns ( 10 feed horns/frequency bands per side) that collect the microwave sky signals focused by the TRS telescopes, as well as the warm microwave components that amplify and detect 


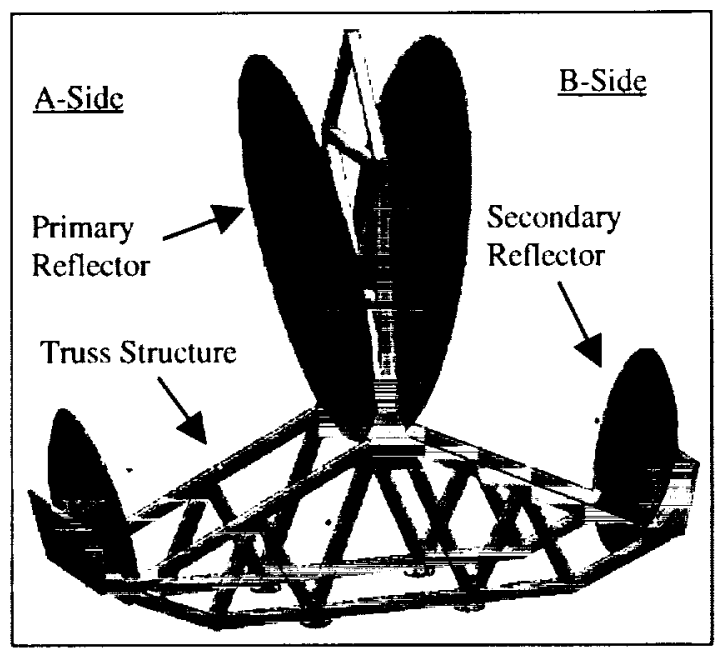

Figure 3 - TRS Structure

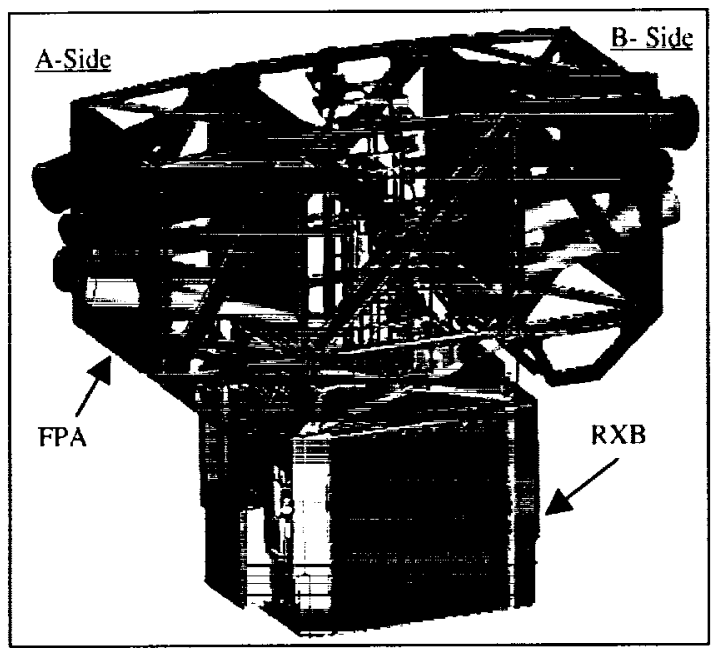

Figure 4 - Microwave System Assembly

these signals. The RXB houses the warm microwave amplifiers. The FPA, RXB, and feed horns are constructed of aluminum. The feed horns feed the signal into copper waveguides and into the RXB.

\subsection{Test Overview}

The primary objective of these measurements was to validate the design displacement predictions of the MAP hardware during thermal vacuum (T/V) testing. Two different test articles, the MS and the TRS, would be tested. This required two separate $T / V$ chamber sessions so that the test articles could be swapped-out. Both of the test sessions were designed to perform the displacement measurements at the mission operational temperature of $\sim 60-90 \mathrm{~K}$.

The primary objective of the MS test article configuration was to validate the feed horn displacement predictions. Secondary objectives were to verify that there were no permanent deformations of the FPA structure, and to observe displacement of targets placed on the Receiver/Transceiver Box (RXB) mounted below the FPA. Deformations on the order of \pm 0.100 inches $(2.540 \mathrm{~mm})$ were expected on the MS test article.

The TRS test article configuration would attempt to validate the TRS reflector displacement and distortion predictions. Secondary objectives were to observe deformations of any FPA portions that were visible to the camera, and to observe flange deformations on certain feed horns to determine if a gap between the flange and inner bulkhead developed as the structures got cold. Deformations on the order of \pm 0.030 inches $(0.762 \mathrm{~mm})$ were expected on the TRS test article.

\subsection{Photogrammetry Description}

The PG system was chosen to perform these measurements because it could be converted to operate in a hands-off capacity. Since the test articles would be enclosed in the T/V chamber during the thermal tests, remote operation was an important capability.

Photogrammetry (PG) is a metrology method that uses digital photographs and the principals of triangulation to obtain its results ${ }^{1.2}$. The PG system consists of the following components:

- INtelligent digital CAmera (INCA) (modified Kodak DCS series camera)

- Laptop with data analysis software (VSTARS) and a network connection to the INCA camera

- Retro-reflective targets \& scale bars of various lengths with a known coefficient of thermal expansion (CTE) 
PG measurements involve taking multiple digital photographs of the test object (targeted with the retroreflective targets) from various positions around the object (to obtain proper triangulation of the measured target points). Triangulation is performed by the software, calculating the position of measured points. Resection is then performed, calculating the position of the camera at the time each picture was taken. The process of triangulation and resection by the software is referred to as a software bundle.

The accuracy of the measurements, and the ability of the software to bundle the shots, is a balance between the targeting scheme (number and placement of targets on the test object), the number of shots (photographs) taken, and the positions from which the shots were taken. The software provides indications of the quality of the job after the bundle is completed. By verifying that these quality values are within an acceptable range, the quality of a PG job can be assured.

Since PG measurements are inherently dimensionless, at least one known distance is required to scale the measurement. Scale bars are placed in the PG targeting scheme to provide this known distance.

\subsection{DEVELOPMENT EFFORTS}

In order to operate the PG camera system remotely, various support equipment had to be developed to allow the PG camera to interface with the T/N chamber and the testing hardware. Remote systems needed to be developed to power, control, house, and move the camera.

The test articles would be surrounded by the helium shroud, which cools the test article during thermal testing. Since the helium shroud completely encloses the test article, the modifications required to accommodate the PG camera system would need to be made to the helium shroud.

The helium shroud structure supports twenty-eight cyropanels, providing a total area of $\sim 300$ square feet to radiatively cool the test article. The structure is octagonal in shape, measuring 110 inches $(2.79 \mathrm{~m})$ across and 182 inches $(4.62 \mathrm{~m})$ high. The cryopanels are plumbed into a recirculating helium skid that is capable of providing over $1000 \mathrm{~W}$ of cooling at $20 \mathrm{~K}$. The shroud is flooded with LN2 during cryogenic operations. The helium shroud crown is lowered onto the helium shroud walls via a crane once the test article has been placed inside the shroud. A cylindrical-shaped thermal blanket is used to closeout the upper section of the helium shroud from the $T / \mathrm{V}$ chamber environment.

All support equipment developed had to be able to withstand and operate under the cryogenic temperatures planned for the test.

\subsection{Camera Power Supply and Network Interface.}

Since the camera was to be enclosed inside the $\mathrm{T} / \mathrm{V}$ chamber and operated remotely during testing, external power and network capabilities were required in order to operate the camera system from outside the T/V chamber. The PG system vendor specially designed and built a power supply/network module to provide an external interface with the camera. The power interface fed the camera and the strobe-flash, while the network connection established a link between the camera and a laptop computer outside the $T / \mathrm{V}$ chamber.

\subsection{Camera Canister Development.}

Since the PG camera system was designed for operation at room temperature, and measurements were to performed with the camera inside the $T / V$ chamber in vacuum and at temperatures of $\sim 60-90 \mathrm{~K}$, a protective environment needed to be developed for the camera. The camera canister was developed to house the camera and provide a stable environment. Essentially a closed cylinder with a viewing window (see Figure 5), the canister provides a pressurized, temperature-controlled environment for the PG camera.

The canister is equipped with heaters and a fan to offset the cryogenic temperatures in the T/V chamber. In order to prevent flashback from the ring strobe (mounted on the front of the camera) off of the outer window surface and back into the camera, the canister window was designed as a two-part window with 


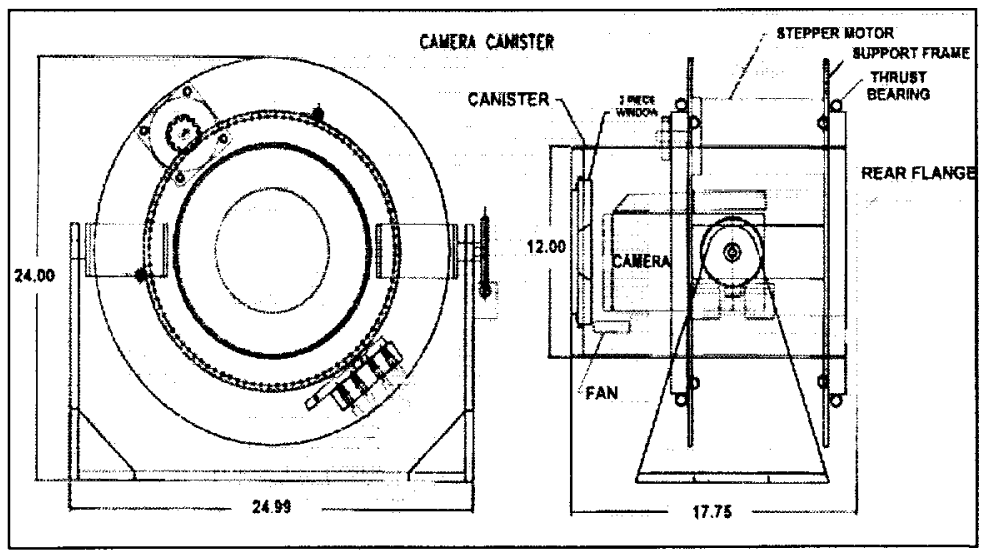

Figure 5 - Camera Canister

conical-shaped bonding. A bulkhead connector on the canister allows the power and network connections to be made to the camera.

The camera canister also provided the ability to rotate the canister 90 degrees about the pointing angle of the camera (shots at 0 and 90 degrees were required to satisfy camera self -calibration requirements). A stepper motor mounted to the canister support frame provided the canister rotation capability.

\subsection{Camera Carousel Development.}

In order to measure a test article with PG, a method for moving the camera to various stations around the test article while inside the $T / N$ chamber was required. The camera carousel, shown in Figure 6, was designed to allow the camera canister to move $\sim 350$ degrees around the perimeter of a test article.

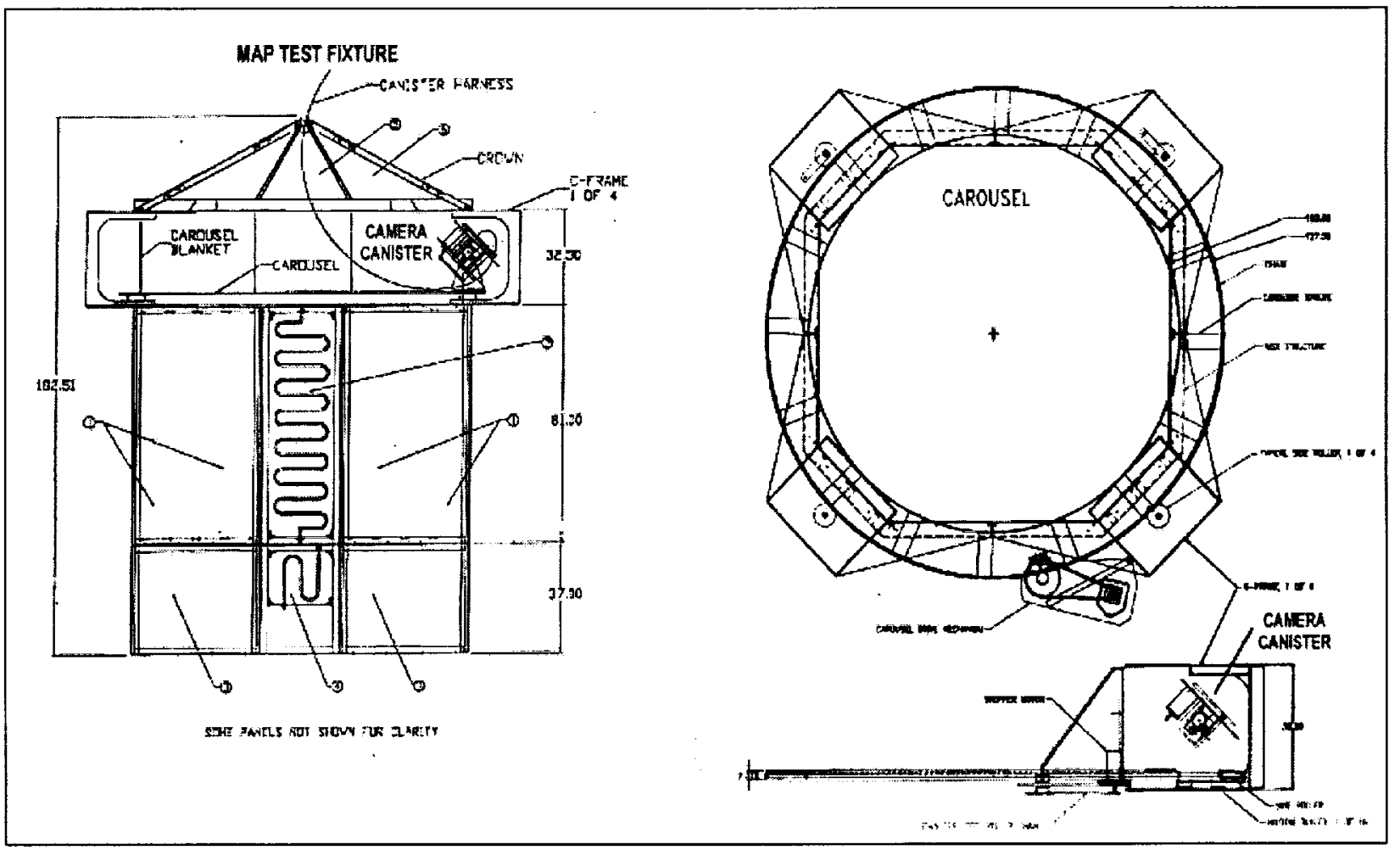

Figure 6 - Canister/Carousel Configuration 
Essentially a circular chain-driven track (measuring $~ 110$ inches $(2.79 \mathrm{~m})$ in diameter), the carousel would be mounted on top of the helium shroud below the shroud crown. The camera canister would be mounted to the track and transported around the test article by the carousel. The carousel would be remotely operated from outside the $\mathrm{T} / \mathrm{V}$ chamber via software control.

\subsection{PG MEASUREMENT METHODOLOGY}

Once PG was chosen as the metrology method and the support GSE was developed to allow PG to be used remotely from outside the $T / V$ chamber, efforts could be focused on how the measurements would be taken. Significant efforts were expended to streamline the metrology procedures and ensure that the measurement methodology was sound, since the measurements would be taken remotely during the $T / \mathrm{V}$ chamber operations. Interruption or delay of a $\mathrm{T} / \mathrm{V}$ test to make changes to the camera system or the targeting would be relatively expensive.

\subsection{Photogrammetric Targeting.}

PG targets are used to define coordinate systems, help the PG software bundle the job, and provide measurement values for points of interest. Development of a good targeting scheme design is driven by the shape and size of the object itself, and the number of stations from which the PG photos will be taken. For complex structures, various viewing angles (elevations) are usually required to see the targets. In the case of the MS and TRS test articles, the camera was restricted to a single plane (defined by the canister on the carousel), and the structures were quite complex. The number of possible carousel stations around the objects were nearly unlimited, but changes in elevation could not be achieved. Proper design of the targeting scheme for the $\mathrm{T} / \mathrm{V}$ test was even more important since access to the test articles could not be gained to add or adjust targets once the $\mathrm{T} / \mathrm{V}$ chamber doors were closed.

The types of targets used in the MS and TRS test article targeting schemes are described below:

- Coded Targets - A coded target consists of a series of retro-reflective dots that are uniquely patterned to make it immediately identifiable to the PG software. The software uses the coded targets to correlate the positions of the targets between photographs, helping it to identify the non-coded targets during the bundling process.

- Retro-Reflective Dot Targets - These targets, approximately 0.25 inches $(6.35 \mathrm{~mm})$ in diameter, were used to target various points of interest on structures.

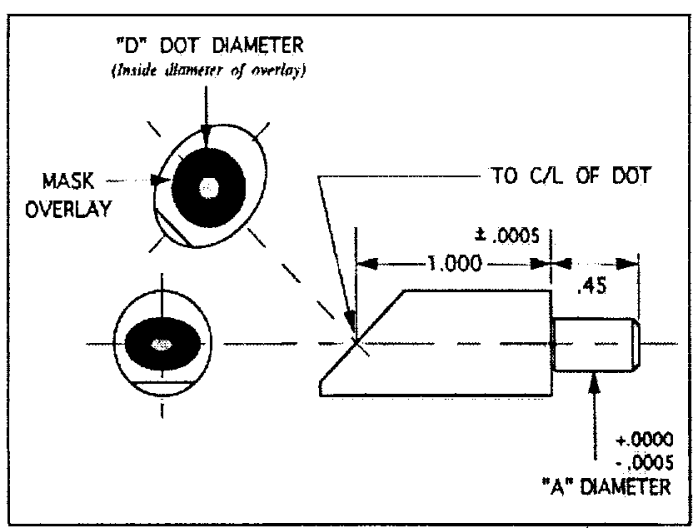

Figure 7 - 45 Degree Angled Target
45 Degree Angled Targets - These targets, shown in Figure 7 , consist of a retro-reflective dot placed on a surface angled 45 degrees from the shank centerline. The angled target and offset these targets provide allow for greater visibility than surface targets. A tooling hole is required in the structure being measured for these targets to be installed.

- Tooling Hole Bosses (THB's) - Not a target itself, a THB is a target holder that has been bonded to the structure being measured. Any variety of targets can be installed into the THB.

Analyses/simulations performed by the PG system vendor helped establish the best regions for targeting in terms of expected accuracy. The final number and location of targets within those regions were determined by GSFC. Test shots of the test article setups were performed to verify the targeting schemes and to build 
the software template for the two test articles. Portions of the final targeting schemes for the MS and TRS test articles are shown in Figures 8 and 9.

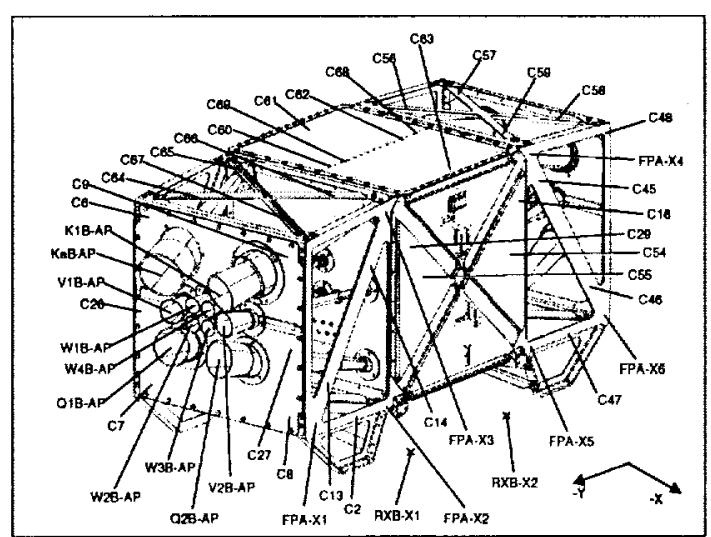

Figure 8 - MS Targeting Scheme

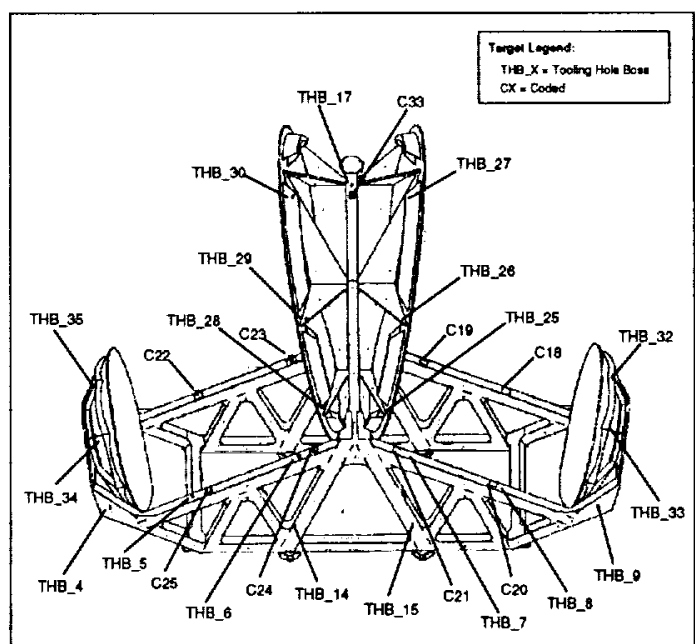

Figure 9 - TRS Targeting Scheme

Two aluminum scale bars were incorporated into each of the test article setups, which the PG software uses to scale the job. Each scale bar provided three scaling distances (six targets). The effects of thermal expansion and contraction of the scale bars needed to be accounted for since the T/V test environment involved radical changes in temperature. A scale factor was calculated for each PG run, knowing the temperature of the scale bars and the CTE of the scale bar material. The test article/scale bars could not be deforming at the time of the PG measurement. A temperature change rate of $\leq 1{ }^{\circ} \mathrm{C}$ per hour was required before a PG run (series of PG shots) could be made. Thermal instrumentation (platinum resistance thermometers were placed on various portions of the test article and on the scale bars) provided real-time temperature data of the test article and the scale bars.. The rate of temperature change was verified before each PG measurement. In addition, the scale bars were thermally isolated from the structure with blocks of $\mathrm{G}-10$ isolation material.

\subsection{PG Software Template.}

Due to the large number of PG runs planned for each test article, attempts were made to minimize the processing time for each run and streamline the bundling process. To accomplish this, a template job was developed for both the MS and TRS test article setups. A template file provides the following advantages:

\section{Automation of Bundling Process:}

When a job is bundled, the software looks for points in the photographs and assigns its own labels to the target points it identifies. Each of these labels must be manually changed to reflect the labels defined by the targeting scheme. Normally, this process would be required for each set of pictures that are bundled. Use of a template job limits this renaming process to only the first bundle. The template job automates the bundling process by storing the name and location of desired targets. When using a template job on a new set of pictures, the software looks for these targets stored in the template, and automatically names them. The template job is created after the labels on the first set of pictures has been renamed to be compatible with the targeting scheme. For measurements requiring multiple PG runs of the same setup (as is the case with the MS and TRS test articles), the use of a template file saves time and effort on the part of the PG operator.

Another advantage of a template is that it forces the software to look only for those points stored in the template file. Without a template, the software searches for all possible targets in the pictures. This can be a problem if there are non-targets such as glints or reflections in the pictures. Any 
errant/mistaken targets would have to be manually removed during the bundling process. Again, use of the template saves time and effort on the part of the operator.

\section{Establishing a Coordinate Reference Frame (CRF):}

When a set of pictures is bundled, a default CRF is generated by the software. The bundle data needs to be transformed from the local CRF to the test article CRF before data can be analyzed. The test article CRF is defined by a subset of the measured points (that have known coordinates in the test article CRF). Once collected, this design data is imported into the software template file. The software then determines a best fit between the measured points and the design data. The end result is a bundled job containing measured points that have been best fit and scaled to the design data.

In the case of the MS test article, 12 points along the sides of the FPA (6 per side) were used as design data and were imported into the software template to define the instrument CRF. These same points had previously been measured and valued in the CRF using two other metrology systems. When the MS PG runs were bundled, the FPA targets were best-fit to the design data. The remaining targets (coded, aperture, RXB, etc.) were allowed to "float" (the software did not include them in the best fit).

For the TRS test article the TRS CRF was defined by a subset of the THB targets mounted on the truss structure. These targets had been previously measured and valued in the CRF by the manufacturer of the TRS structure and GSFC using various metrology systems. The manufacturer data was imported into the software template for the TRS test article as design data. As with the FPA test article measurements, the remaining targets (coded, FPA, reflector, etc.) were allowed to float.

The development and use of a software template file for the MS and TRS test articles successfully automated the processing of data for each of the PG runs, significantly reducing the amount of time required to bundle each run.

\subsection{Error Analysis.}

An error analysis was required to ensure that the measurement requirements for the two parts of the $\mathrm{T} / \mathrm{V}$ test were met. It was determined that the camera system itself and the targeting were the major sources of error for these measurements.

The measurement precision of the photogrammetric camera is \pm 0.002 inches $(0.05 \mathrm{~mm})$ at a range of $8 \mathrm{ft}$. $(2.44 \mathrm{~m})(2 \sigma)$. The separation distance between the camera and the targets was less than $8 \mathrm{ft}$. for all of the pictures taken in the $\mathrm{T} / \mathrm{V}$ chamber.

The PG camera is self-calibrated as a byproduct of the measurement. There are three requirements to achieve self-calibration. The first is to roll the camera 90 degrees for half of the pictures taken. Second, there is a minimum number of pictures that must be taken from a minimum number of locations. Third, there is a minimum number of well-distributed targets visible in each picture and for the entire test article. All of these requirements were addressed during the development of GSE and the targeting scheme for the T/V tests.

Through the process of bundling, the PG software provides indications of job quality and the occurrence of errors. By carefully examining the software quality/error indicators and through proper use of the camera, the worst-case error contribution from the PG camera itself is $\leq \pm 0.002$ inches $(0.051 \mathrm{~mm})$

Target placement and target repeatability errors can be attributed to the PG targets used on the FPA during the T/V test. The overall target error contribution (a combination of the these two errors) was calculates to be $\leq \pm 0.0015$ inches $(0.038 \mathrm{~mm})$.

Taking all of the above errors into consideration, the total error for a given PG run can be established to be $\leq \pm 0.0035$ inches $(0.089 \mathrm{~mm})$. This ensures that all measurements will be able to resolve the $\pm 0.100 \mathrm{inch}$ 
$(2.54 \mathrm{~mm})$ and \pm 0.030 inch $(0.762 \mathrm{~mm})$ deformations that were predicted for the MS and TRS test articles, respectively.

\subsection{PG MEASUREMENTS/ANALYSIS}

A PG run consisted of a series of pictures taken at various carousel stations around the test article. Two pictures were required at each station to facilitate the camera self calibration - one with the canister at 0 degrees and the other with the canister at 90 degrees. The number and location of carousel stations was predicted and tested during the pre-chamber targeting verification tests.

For each test article, the first PG run in the T/V chamber was performed at ambient temperature and pressure to establish a pre-test baseline and to verify the carousel stations. As the first few PG runs progressed, the number of carousel stations and their locations around the test article were modified slightly to optimize the camera's view of the test article targeting.

\subsection{PG Runs.}

The final carousel station layout for the MS and TRS test articles are shown in Figures 10 and 11. A total of 44 stations were used during the MS test article PG runs, resulting in 88 PG shots (camera at 0 and 90 degrees at each station). The TRS test article PG runs consisted of 42 carousel stations, resulting in 84 PG shots. It took the camera operator and carousel operator $\sim 30$ minutes to obtain all of the shots in a PG run.

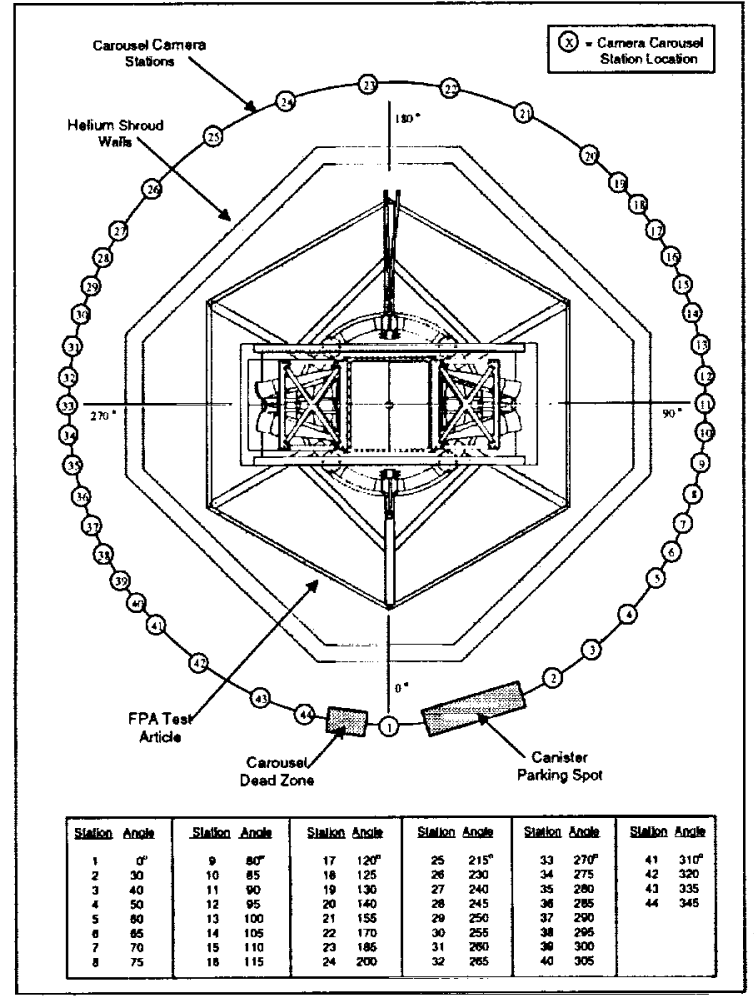

Figure 10 - MS Carousel Layout

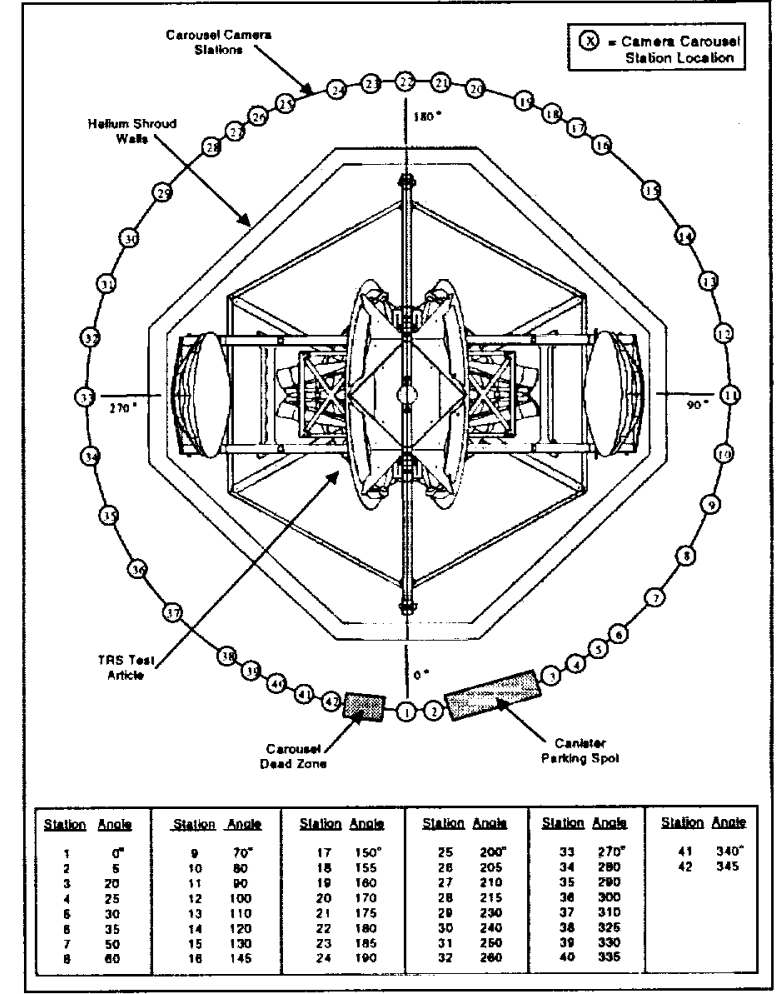

Figure 11 - TRS Carousel Layout

Once the PG shots for a given PG run were taken, the run was bundled using the template job. Once the bundle was deemed successful $(\sim 20$ minutes of processing and analysis time by the operator on the laptop computer), the PG operator would give concurrence for the test to proceed to the next test (temperature) point. 
A series of PG runs had been planned for the T/V testing as a baseline. However, the number of PG runs and their timing was changed as the T/V testing progressed. A total of six PG runs were made for the MS test article, while five PG runs were made for the TRS test article.

Each MS and TRS test article PG run was bundled using the template to automate the job bundle. Each job was transformed into the appropriate coordinate reference frame via a best fit with design data (present within the job template). An EXCELTM program was used to determine the scale factors and CTE for the scale bars at each temperature case. Once the job was scaled, the measured points were exported to an EXCEL ${ }^{\mathrm{TM}}$ program in order to compare the runs. Data tables were developed to present the results of the measurements. For each of the two parts of the test, each of the PG runs was compared to all of the other runs to determine the target displacements from case-to-case.

\subsection{PG MEASUREMENT RESULTS.}

Both the MS and TRS test articles were thermally tested in the T/N chamber at cryogenic $(\sim 60-90 \mathrm{~K})$ temperatures. PG measurements were successfully made using the PG system remotely from outside the $\mathrm{T} / \mathrm{V}$ chamber. Brief summaries of the results of these measurements are provided in the sections below.

\subsection{MS Test Article Results.}

The primary measurement objective of the MS test article configuration was to validate the feed horn displacement predictions. Secondary objectives were to observe any displacement/rotation of the RXB and to verify that there were no permanent deformations of the FPA structure.

The following result summaries were derived from the data obtained during the six PG runs of the MS test article:

\section{Entrance Aperture Translations:}

The feed entrance aperture translations were all within 10 percent of predictions. Translations were on the order of +0.100 inches.

\section{RXB Motion:}

RXB motions were determined by least squares fitting the warm positions to the cold positions. The resulting translations and rotations of the RXB were as expected, based on hand calculations.

\section{Post-Test Residuals:}

The final ambient measurement data was compared to the initial ambient measurement data to identify residual deformation of the structure. There were no apparent residual deformations due to thermal testing, based on a comparison of 164 targets. The standard deviations for residuals in each coordinate axis werc 0.001 inches $(0.025 \mathrm{~mm})$. The maximum residual was 0.004 inches $(0.102 \mathrm{~mm})$. The estimated PG measurement error is \pm 0.0035 inches $(0.089 \mathrm{~mm})$.

\subsection{TRS Test Article Results.}

The primary measurement objectives of the TRS test article configuration were to validate the TRS reflector displacement and distortion predictions and derive a CTE value for the composite truss structure. Secondary objectives were to validate the gapping between the feed flanges and the FPA inner bulkhead and to verify that there were no permanent deformations of the TRS structure.

The following result summaries were derived from the data obtained during the five PG runs of the TRS test article: 


\section{Reflector Deformations/Position:}

The measured reflector deformations confirmed measurements that were made previously on the Reflector Engineering Unit (REU). Due to difficulties in accurately modeling the complex curved honeycomb composite reflectors, measurements at temperature were relied on to determine the shape deformations. Consistency in the results provided confidence in the shape values. Overall deformations, removing rigid body motion, were on the order of \pm 0.030 inches for the primary reflectors and \pm 0.015 inches for the secondary reflectors.

Reflector position changes due to cool-down revealed a discrepancy in the truss CTE values. The secondary reflectors translated inward, where they were predicted to move outward, due to a negative truss CTE.

\section{Truss CTE:}

The apparent CTE of the truss structure was $+4.9 \mathrm{E}-7 \mathrm{in} / \mathrm{in} / \mathrm{K}$, based on the PG measurements. The predicted CTE based on laminate properties was $-5.1 \mathrm{E}-7 \mathrm{in} / \mathrm{in} / \mathrm{K}$. Although uncertainty was later placed on both CTE values due to simplifying assumptions, the test revealed secondary reflector translations to be opposite of that predicted and ultimately resulted in slight repositioning of replacement secondary reflectors.

\section{Flange gapping:}

Targets were added to the FPA structure for measuring flange gapping during the TRS testing. Gaps of $0.002-0.006$ inches were measured. The values compared well with the 0.005 inches predicted for the 4 feeds measured.

\section{Post-Test Residuals:}

The final ambient measurement data was compared to the initial ambient measurement data to identify residual deformation of the structure. There were no apparent residual deformations due to thermal testing, based on a comparison of 185 targets. The standard deviations for residuals in each coordinate axis were 0.002 inches $(0.051 \mathrm{~mm})$. The maximum residual was 0.007 inches $(0.178 \mathrm{~mm})$. The estimated PG measurement error is \pm 0.0035 inches $(0.089 \mathrm{~mm})$.

\subsection{CONCLUSION}

The PG measurements under cryogenic T/V chamber conditions were a success based on the data that has been analyzed and presented. The concept and design of the camera canister and carousel GSE worked well, providing a flexible metrology platform for the PG camera. The targeting schemes that were developed for each test article provided visibility of the regions in-question, as well as the accuracy required to measure their distortions.

With the combined MS and TRS PG runs measurements were obtained for feed translations, flange bulkhead gapping, RXB motion, reflector deformations, and reflector motion resulting from transition to cryogenic temperatures. These measurements were successfully made in parallel with the thermal performance test objectives.

Overall, the MAP optical measurement test objectives were met using these techniques in the thermal/vacuum environment. 


\section{ACKNOWLEDGEMENTS}

The authors would like to thank the PG System vendor, Geodetic Services Inc., for their hardware design and development effort, as well as their support in getting the most out of the measurements. Our thanks also go out to Mantech Services Inc. for the technical support in preparing and 24 hour operation of the T/V

chamber, to Dean Osgood of Mantech Services Inc. for the theodolite, laser tracker, and photogrammetry measurement support, and to our Mechanical Systems Analysis \& Simulation group for mechanical systems analysis.

\section{REFERENCES}

1. Elements of Photogrammetry, Paul R. Wolf, McGraw Hill 1983

2. Automation in Digital Close-Range Photogrammetry, Clive S. Fraser, First Trans Tasman Surveyors Conference, 12-18 April, 1997, New Castle 